\title{
POR UNA ESCUELA VIVIDA COMO COMUNIDAD EDUCATIVA
}

\section{For a School Lived as an Educational Community}

\author{
Emanuele BALDUZZI \\ Instituto Universitario Salesiano de Venecia. Venecia. \\ e.balduzzi@iusve.it
}

https://orcid.org/0000-0002-6053-6515

Fecha de recepción: 01/09/2020

Fecha de aceptación: 05/11/2020

Fecha de publicación en línea: 01/03/2021

Cómo citar este artículo: Balduzzi, E. (2021). Por una escuela vivida como comunidad educativa. Teoría de la Educación. Revista Interuniversitaria, 33(2), 179-194. https://doi. org/10.14201/teri.23774

\section{RESUMEN}

Este artículo propone destacar las potencialidades educativas propias de la escuela vivida como una comunidad educativa. Para lograr tal cosa, toda escuela debería asumir el sentido más profundo de su etimológia griega: scholé, es decir «tiempo libre», desligado de las actividades productivas, y por esta razón empleado para la formación personal. Esta ha de defender y promover una mejora armónica y global de la persona que, en una auténtica comunidad educativa, se fomenta: 1) al promover y cuidar los «bienes relacionales», no solo los «convergentes»; y 2) mediante las vinculaciones y afectos "constitutivos». Los primeros bienes citados solo pueden existir si se dan una relación interpersonal y un vínculo que los vivifiquen, en cuanto son bienes compartidos e indivisibles, necesariamente procedentes del entorno social. Por su parte, los afectos y las vinculaciones "constitutivas", que son la base de una "comunidad constitutiva", resultan fundamentales para la construcción de nuestra identidad, pues muestran el valor antropológico y educativo de las relaciones interpersonales que establecemos. En particular nos ofrecen la oportunidad de comprender y apreciar la interdependencia positiva que surge en un contexto social compartido en virtud de 
un bien relacional que protegemos y cuidamos. La sección conclusiva quiere mostrar que la búsqueda del bien constitutivo es esencial para que una escuela comunitaria no caiga en la manipulación y la homologación.

Palabras clave: escuela; comunidad educativa; scholé; bienes relacionales; emociones.

\section{ABSTRACT}

The main goal of this article is to highlight the didactic power of an educational community. In order to perform this role, schools should pursue the deeper, etymological meaning of the word itself: schole as leisure time, free of materially productive activities, therefore dedicated to personal growth. The school's highest task consists in defending and supporting the global, balanced development of students in an authentic educational community, first by appreciating relational skills, not only performing ones, and second by promoting essential relationships and bonds. These can only exist in a personal relationship and in a bond that strengthens them, since they are meant to be shared and cannot be divided, but rather live only in a social context. Moreover, "essential» emotional bonds, which are pivotal in an essential community are necessary for the development of one's identity, since they show the anthropological and didactic value of the interpersonal relationships that are their own essence. In particular, they give us the opportunity to understand and appreciate the positive interdependence that is born in a shared social context, founded on a relational value that is protected by its members. The conclusion is focused on the necessity of the pursuit of an essential value in a school considered as a community, which rejects standardizing and manipulation.

Key words: school; educational community; scholé; relational goods; emotions.

\section{INTRODUCIÓN}

La escuela es un ámbito de "gran relieve educativo» (Mari, 2017) para miles de personas, sobre todo durante los años 6-16, que suelen coincidir en muchos países con la enseñanza obligatoria. En este sentido, «un tesoro para salvar» (Genovesi, 2020), un «bien común» (Triani, 2020) que —en cuanto a tal - tenemos que cuidar y proteger (Balduzzi, 2012). Por ello, durante estos últimos meses, a raíz del cierre gubernativo de todas las escuelas en el territorio italiano (desde finales de febrero 2020), diversos profesores y alumnos, y también miembros de la sociedad civíl, han subrayado la importacia, la centralidad y la transcendencia de las instituciones educativas formales, cuya vertiente educativa no se agota en el mero proceso de enseñanza-aprendizaje (Biesta, 2012, p. 583).

Por lo dicho, creo que el desafío de mayor calado hoy es definir cuál ha de ser el verdadero núcleo formativo de la escuela, pues nos enfrentamos a una encrucijada (Baldacci, 2019): por un lado, está el mercado, con sus exigencias de competencia 
técnica y eficiencia; y, por el otro, la democracia, con su ideal de emancipación humana. Dicho de otra manera: una escuela organizada "como aparato", con un sistema de reglas rígidas y uniformes de alcance nacional y a veces incluso internacional; o una escuela al servicio de su más propia misión educativa, que es la promoción de la libertatad y la responsabilidad personal (Magni, 2020), lo que la capacitaría para convertirse en una «formidable argamasa civil, social, ética y cultural.» (Bertagna, 2020, p. 119).

Solventar tal reto implica tomar plena conciencia de lo que es verdaderamente relevante con vistas al perfeccionamiento de los alumnos. Y para cumplir en esta finalidad, toda escuela debería retomar el sentido más profundo de su etimológia griega: scholé. Como nos recuerda Giuseppe Mari (2014, p. 10):

Tanto las palabras de las lenguas romances que designan la escuela (además del italiano scuola, podemos recordar école en francés, escuela en español, escola en portugues, şcoală en rumano), como las de las lenguas nórdicas (school en inglés, Schule en alemán, skola en sueco...) vienen de una palabra griega —scholé - que significa 'tiempo libre' [...] es decir «tiempo libre» de actividades productivas, y por esta razón empleado para la formación personal.

La enseñanza debe permitir alcanzar nuestra plenitud humana a través de un proceso formativo que nos convierta en seres humanos libres, en lugar de inclinar a los alumnos tan solo a adquirir destrezas (skills) tecnicas u habilidades operativas y productivas (Mari, 2014). De hecho, como subrayan Masschelein y Simons (2014, p. 3):

Desde su origen en las ciudades-estado griegas, la escuela fue una fuente de 'tiempo libre’ - la traducción más común de la palabra griega scholè- para el estudio y para la práctica concedido a personas que no tenían derecho a él (según el orden arcaico que prevalecía en la época). Así pues, la escuela era una fuente de conocimiento y de experiencia disponibles como 'bien común' [...].

Creemos que es precisamente hoy - en el momento en que muchos la condenan por inadaptada a la realidad moderna y otros incluso parecen querer abandonarla completamente- cuando aparece explícita y claramente lo que la escuela es y lo que hace. También confiamos en dejar claro que muchos de los alegatos contra la escuela están motivados por un antiquísimo temor (e incluso por el odio) a una de sus características más radicales pero que la definen esencialmente: que la escuela ofrece 'tiempo libre', que transforma los conocimientos y destrezas en 'bienes comunes' y, por lo tanto, que tiene el potencial para proporcionar a cada cual, independientemente de sus antecedentes, de su aptitud o de su talento natural, el tiempo y el espacio para abandonar su entorno conocido, para alzarse sobre sí mismo y para renovar el mundo (para cambiarlo de un modo impredecible).

Este principio - fundamental en el pensamiento filosófico-pedagógico occidental-, hoy en día parece olvidado, no tanto en las reflexiones teóricas, sino más bien en la práctica docente. Por ejemplo, en Italia, toda escuela debe definir su POF, es decir el Piano dell'offerta formativa, que, como se lee en la página web 
del Ministero della Pubblica Istruzione, "es el DNI de cada escuela»: determina sus rasgos distintivos, el proyecto pedagógico-cultural que la anima, su diseño curricular y extracurricular, la didáctica y la organización de sus actividades (Balduzzi, 2015, p. 123).

En el POF, muchas veces, se incluyen metas como la tolerancia, el respeto, la responsabilidad, el desarrollo libre y completo de almunas y alumnos. Sin embargo, en la práctica y en el día a día, los aspectos técnicos, la certificación de competencias, los criterios de evaluación y la complejidad de los procesos burocráticos eclipsan los ideales que deberían vivificar la actividad escolar. Sin embargo, como afirma Trilla (2004, p. 321):

Es precisamente en la posibilidad de desmarcarse parcialmente de los valores o contravalores dominantes en el medio donde reside la educatividad ética y cívica de la escuela. Si afirmamos que la escuela puede y debe desarrollar un papel — compartido, pero remarcable- en la formación en valores y en la educación de la ciudadanía, estamos afirmando a la vez la capacidad que tiene la escuela de hacer una cierta criba de valores, deposicionarse frente a ciertos contravalores de su entorno social. Para limitarse transmitir los valores que, para bien o para mal, imperan en el medio la acción de la escuela sería innecesaria.

No se pretende despreciar las competencias y las evaluaciones, que son necesarias en un contexto educativo formal, incluidas las competencias cívicas, con sus derivaciones éticas, que son esenciales para todas las sociedades democráticas (Mari, 2018, pp. 46-47), cuya meta es buscar el «bien» para uno mismo y los demás (Sandrone, 2018), y que, como nos recuerdan los documentos internacionales, han de ser practicadas durante toda la vida, y ejercidas por todos y, empezando por las escuelas (Recomendación del Consejo de Europa del 22 de mayo del 2018 sobre las competencias clave para el aprendizaje permanente) $)^{2}$.

Sin embargo, no podemos olvidar que no basta con citar bienes educativos o simplemente indicar valores (Mari, 2013), aunque tengan gran calado y mucha relevancia, en un documento escrito o una página web (por más que se le otorgue un carácter "oficial»), sino que se impone crear también espacios y momentos que permitan su asunción y aplicación, lo que implica idear concretas para los alumnos y para los profesores. Como nos recuerda Puig (2012, p. 101):

Los centros educativos deben esforzarse por alcanzar los niveles de exelencia que marca la consecución de este bien que los define. Para lograrlo deben istituzionalizar este bien. No es suficiente con proclamarlo, sino que deben buscar las formas institucionales que permitan proporcionarlo con la máxima solvencia a los jóvenes.

1. https://archivio.pubblica.istruzione.it/argomenti/autonomia/pof/default.shtml

2. Consejo de Europa (2018). Recomendación del Consejo del 22 de mayo del 2018 sobre las competencias clave para el aprendizaje permanente. https://eur-lex.europa.eu/legal-content/IT/TXT/ PDF/?uri=CELEX:32018H0604(01) 
No olvidemos que, como nos enseña el modelo finlandés (Sahlberg, 2011b), que es posible obtener buenos resultados docimológicos —incluso en los estudios OCSE-PISA (Sahlberg, 2011a) — sin prestar demasiada atención a cuestiones instrumentales, como el rendimiento de cuentas y los controles periódicos de evaluación, si existe un fuerte ideal educativo compartido, si hay metas que se alcanzan todos juntos, confiando en los profesores y en su capacidad de innovación y desarrollo humano y profesional (Hargreaves \& Shirley, 2009).

A raíz de lo expuesto, surgen las siguientes preguntas: ¿cómo y en qué medida interactúan en la escuela el ideal educativo y su traducción concreta?, y por otra parte, ¿debe buscarse que los estudiantes alcancen su plenitud personal, o basta con que adquieran habilidades técnicas e instrumentales?

\section{UNA ESCUELA VIVIDA COMO UNA COMUNIDAD}

Para implantar, y al mismo tiempo promover, a un ideal educativo necesitamos este tipo de escuela, cuyas características iremos detallando. Solo una escuela vivificada por la identidad colectiva que los Communitarians ${ }^{3}$ han subrayado ofrece la oportunidad de alcanzar y promover metas educativas de relieve (Balduzzi, 2018). Como subrayan Amilburu, Bernal y González Martín (2018, p. 111): «Repensar la escuela supone también interpretarla como generadora de amistad, sentido moral y justicia, en la medida en que es constructora de vínculos personales, comunitarios y sociales». Además, una escuela como comunidad educativa influye de manera decisiva en el crecimiento positivo de alumnos y alumnas (Malaguti, 2016).

Thomas Sergiovanni (2000) nos recuerda que hay una notable diferencia entre una escuela considerada como organización y la otra vivida como una comunidad. Según este autor, en la primera las relaciones interpersonales están determinadas por un vínculo contractual o legal, en virtud del cual se buscan objetivos individuales negociando premios y recompensas. Se pueden pretender también ojetivos comunes, pero siempre bajo una perspectiva instrumental. Este contexto no favorece el perfeccionamiento armónico y global de la persona, pues se orienta hacia la mejora funcional e instrumental de las capacidades y habilidades (olvidando también el sentido de escuela como scholé). Ahora bien, si pretendemos educar en plenitud, deberíamos tener muy presente la elección de los medios con vistas a los fines, para evitar caer en un gravísimo error: el «olvido y la equivocación sobre los fines", como nos recuerda Maritain (2001, pp. 61-63). De hecho, podemos perder la capacidad de formular preguntas profundas sobre la enseñanza si nos centramos solo en la "gestión» del conocimiento (Mínguez Vallejo, 2010, p. 59).

En cambio, en una escuela vivida como comunidad, afirma Sergiovanni (2000), 1) las interdependencias son sentidas; 2 ) hay una vinculación profunda entre las

3. Una exposición precisa y detallada puede hallarse en Naval (2000), Mincu (2007) y Ferrara (1992). 
personas; 3) se promueve y desarrolla una idea construida todos juntos. Además, sostiene Strike (2000), dicha institución se basa en determinados presupuestos. Entre los que destaca, uno me parece particularmente relevante: se comparte una visión de "buena educación» que anima a las personas a unirse para lograrla. Por eso, la verdadera reflexión pedagógica se ha de considerar, no solo la escuela como un sistema integrado por elementos que se influyen entre sí, sino también como comunidad educativa, como un entramado de personas que buscan un fin educativo común.

La pregunta clave, por tanto, es la siguiente: ¿cuáles son los rasgos distintivos de la escuela pensada como comunidad educativa?

\section{LA ESCUELA COMO COMUNIDAD EDUCATIVA}

Antes de entrar en materia, parece imprescindible precisar una cuestión esencial: lo que proponemos es muy exigente, e implica un gran esfuerzo y participación por parte de profesores, padres y alumnos ${ }^{4}$. No obstante, hoy en día parece estar retornando hasta cierto punto este ideal pedagógico-educativo, también para el Ministerio de la Pública Instrucción italiana. Por ejemplo, en un importante documento que hizo público durante el cierre de todas le escuelas italianas: la nota oficial 388 del 17 de marzo del $2020^{5}$. Era la primera y muy esperada norma relativa a la enseñanza a distancia. Al principio de ella (p. 2), se lee lo siguiente:

La enseñanza a distancia, en estos difíciles momentos, ha tenido y tiene dos sentidos. Por un lado, reclama del conjunto de la comunidad educativa, en el marco de las responsabilidades profesionales y, ante todo, éticas de cada cual, continuar desempeñando el cometido social y formativo de 'hacer escuela', aunque no sea 'en la escuela', y de hacer, precisamente, 'comunidad'. Mantener viva la comunidad de la clase, de la escuela y el sentido de pertenencia, combatir el peligro del aislamiento y la desmotivación. Las interacciones entre los docentes y los alumnos pueden ser el aglutinante que preserve y refuerce el entramado de relaciones, la involucración en el desafío que se plantea y el empeño de afrontar una situación imprevista.

Como se ve, en unas pocas líneas, la palabra "comunidad" aparece reiteradamente: comunidad educadora ${ }^{6}$, comunidad escolar, comunidad de clase, "hacer» y

4. Como señala Ulivieri (2018, p. XXIII), «se ha creado una nueva sensibilidad comunitaria que se atrave a dialogar con la libertad individual y colectiva inserta en un contexto regulado, democrático y civil».

5. Miur (2020a). Emergenza sanitaria da nuovo Coronavirus. Prime indicazioni operative per le attività didattiche a distanza. https://www.miur.gov.it/documents/20182/0/Nota+prot.+388+del+17+ma rzo+2020.pdf/d6acc6a2-1505-9439-a9b4-735942369994?version=1.0\&t=1584474278499

6. Esta expresión reaparece en otro documento reciente con indicaciones sobre la reapertura de las escuelas, donde se aboga por una alianza entre las escuelas y las comunidades locales mediante "Pactos educativos de comunidad». Miur (2020b). Adozione del Documento per la pianificazione delle attività scolastiche, educative e formative in tutte le Istituzioni del Sistema nazionale di Istruzione per l'anno 
"mantener viva" a la comunidad. No es algo casual. No podemos olvidar que con tal recomendación buscaba ofrecer una alternativa formativa durante un momento muy difícil y complejo para estudiantes, docentes, familias. También el Presidente de la República italiana, Sergio Mattarella, durante el discurso de apertura del año escolar el 14 de septiembre 2020 a Vo' (Padua) subrayó la importancia de una escuela como comunidad? .

En conexión con lo anterior, a continuación, quiero reflexionar sobre la naturaleza de una escuela vivida como comunidad educativa, centrándome en dos de sus rasgos que son esenciales: 1) la promoción del cuidado de los «bienes relacionales", no solo los "bienes convergentes"; y 2) la existencia de vinculaciones y afectos "constitutivos".

\subsection{Escuela como comunidad educativa y los bienes relacionales}

Antes de empezar, parece imprescindible precisar algo esencial para comprender el sentido de nuestra argumentación: es el bien lo que nos hace percibir algo como deseable y no lo contrario. El bien vivifica el valor convertiendolo también en un bien educativamente relevante. Para identificar el bien que define una escuela en cuanto comunidad educativa nos fijaremos en una tipología específica de bienes: los relacionales, y en particular, los inmediatos compartidos. A este respecto Charles Taylor (1989) nos recuerda que un bien inmediato compartido, como la amistad, no puede equipararse con un bien simplemente convergente, como, por ejemplo, el transporte colectivo. Es decir, algo positivo que por mi cuenta no puedo conseguir, pero si tuviera la posibilidad de hacerlo, eso no afectaría a su carácter ontológico. En el ámbito de la enseñanza, la certificación del dominio de un idioma resultaría equiparable y sería un bien convergente. No podemos conseguirla individualmente, pues necesitamos una escuela que nos la ofrezca, nos admita, nos prepare y nos examine. No obstante, si existiera algún modo de obtenerla en un hipotético centro autorizado con un solo alumno, en nada cambiaría eso al bien mismo poseído, lo que por otra parte lo convierte en instrumental.

Sin embargo, deberíamos tener siempre muy presente que existen bienes que creamos y poseemos en común, resultan indivisibles, necesariamente han de surgir en una comunidad (Taylor, 1985). Por ejemplo, la amistad, precisamente por su propia naturaleza, solo puede darse en el marco de una relación interpersonal y gracias a un vínculo que la vivifica.

scolastico 2020/2021. https://www.miur.gov.it/documents/20182/2467413/Le+linee+guida.pdf/4e4bb4111f90-9502-f01e-d8841a949429

7. Presidenza della Repubblica italiana, Intervento del Presidente della Repubblica italiana Sergio Mattarella all'inaugurazione dell'anno scolastico 2020/2021. https://www.quirinale.it/elementi/50200 
Pues bien, un bien relacional que en la escuela ha de ser promovido y cuidado es la construción compartida del proceso cultural.

Para caracterizarlo partimos de la definición de 'cultura' que encontramos en el Diccionario Treccani ${ }^{8}$ :

El caudal de saberes teóricos que una persona ha adquirido gracias al estudio o la experiencia, reelaborados no obstante tras una reflexión personal y profunda hasta convertir los conceptos de la simple erudición en elementos constitutivos de su personalidad moral, de su espiritualidad y de su sensibilidad estética, en suma, en conocimiento de sí mismo y de su propio mundo.

Como se observa, la cultura implica que los conocimientos, el estudio y las nociones han de ser transformadas y reelaboradas por quien la posee, asumiéndolas personal y críticamente, de modo que se conviertan en elementos constitutivos de su personalidad moral y espiritual, su sensibilidad estética y su autoconciencia. Por otra parte, los conocimientos y las ideas pueden ser valorados y usados también como bienes instrumentales en lugar de convergentes: por ejemplo analizar en profundidad una obra literaria solo para sacar una muy buena nota, o interesarse a la explicación del profesor pues facilita afrontar con garantías el examen. Por supuesto, si fuese posible alcanzar todo esto de manera subjectiva y aislada no tendría sentido ir a la clase. Lo hago solo porque estoy obligado por la ley y por otros motivos ya explicados.

En cambio, si se considera fundamental transformar el contenido de las clases en conocimientos profundos sobre sí mismo y los demás, sobre el mundo complejo en el que vivimos, ese proceso implica una vinculación más profunda, no solo con los profesores, sino también con los compañeros y compañeras. Entonces, lo que se puede lograr reflexionando y aportar a la comunidad de clase cobra fuerza gracias a los esfuerzos, las palabras, la escucha y el apoyo de todos, y se inicia un proceso, no solo de maduración individual, sino también de desarrollo cultural común, si todos consideran esencial la mejora y el aporte personal que cada uno ofrece a los demás y viceversa.

Así pues, el proceso cultural que se promueve y alienta en una clase vivida como comunidad educativa es verdaderamente como un bien relacional, pues no se da sin un contacto profundo y sin la participación de todos. La comunidad de clase comparte y construye la bien cultural mediante las aportaciones que se dan "en común». Eso implica que nadie ha de ser dejado a un lado, ni puede ignorarse ninguna acción, palabra o intervención, si contribuye a ese bien cultural, y al mismo tiempo para mi perfeccionamiento y el de todos los demás.

Este alcance implíca dificultad y complejidad, y también un fuerte compromiso personal. Sin embargo, poner en marcha un proceso educativo de tal calado

8. http://www.treccani.it/vocabolario/cultura/ 
puede constituir el principal reto de la escuela en los proximos años. Configurar la escuela como una comunidad educativa — no tanto burocrática y organizativasupone redefinir los marcos de referencia y de comprensión del mismo concepto de escuela, educación y formación. De hecho, los conocimientos, competencias y destrezas - pilares de la escuela actual - no se pueden convertir de inmediato en bienes relacionales. Por ejemplo, si puede desear poseer muchos conocimientos para sentirse superior a otros compañeros. Pero, ¿por qué un alumno/alumna debería abandonar tal aspiración personal si no se siente vinculado/vinculada de algún modo a ellos?

En cambio, en una auténtica comunidad educativa el bien que se posee en común está constituido por las mutuas aportaciones con vistas al bien de todos, que generan la maduración personal y la edificación compartida de una realidad cultural. Además, se valora la mejora integral de la persona y se impulsa un tipo de cultura que implica la participación y la contribución de todos los alumnos y profesores. No es posible centrarse solo en los contenidos o en la certificación de competencias, aunque estas se definan con esmero y atención para orientar el perfeccionamiento armónico de los estudiantes, pues la plena realización de ser humano se despliega en un horizonte de sentido que construimos, tenemos y cuidamos en común, y solo en común, en cuanto es un bien relacional, puede sobrevivir, donarse, hallarse.

A raíz de lo expuesto, se plantea otra pregunta: ¿Durante esa construcción compartida de la cultura y la formación completa de las personas, qué tipo de vinculaciones y afectos brota?

\subsection{La escuela como comunidad educativa: las vinculaciones y los afectos consti- tutivos}

Como nos recuerdan Iafrate y Bertoni (2010, p. 8), por su raíz etimológica, el término "afecto" implica "ser impresionado', 'ser movido' por alguien; ante todo, es un encuentro con el otro; algo o alguien me impresiona y yo me voy hacia él». Por eso, las emociones nos muestran lo que tiene particular sentido y valor para nuestra vida, y también en la escuela, tiñen de "color antropológico» de nuestras relaciones interpersonales. Además, explica Nussbaum (2001), cada emoción conlleva creencias complejas sobre un objeto en particular. Por ejemplo, volviendo al ámbito que nos ocupa, si creo que mi contribución será determinante para la maduración de mis compañeros, surgirán emociones derivadas de esa creencia. Por otra parte, los afectos, es decir, el conjunto de las emociones, sentimientos, pasiones, sentidos vitales... (Rossi, 2002), fortalecen o debilitan las relaciones interpersonales, las contribuciones compartidadas, las interdependencias en el aula.

Pues bien, una dimensión esencial de la escuela que se propone en este artículo subraya, por un lado, la comunidad de emociones y los afectos "constitutivos", y el descubrimiento de las vinculaciones constitutivas, por otro. 
Como se ha explicado, en ella el bien relacional se dona a todos porque es educativo, pues contribuye tanto a la maduración y la mejora personal como al proyecto cultural común. Cabe por ello plantear esta pregunta: Supuesto ese bien relacional, ¿cómo cambian ontologícamente las relaciones interpersonales, los afectos y las vinculaciones profundas?

Los afectos y las vinculaciones "constitutivas", que permiten consolidar una "Comunidad constitutiva" (Sandel, 1994) son fundamentales para la construcción de nuestra identidad, pues nos ayudan a captar la relevancia antropológica y educativo de nuestras relaciones interpersonales. En particular, nos permiten comprender y apreciar la positiva interdependencia que surge en un contexto social compartido, cuyo fundamento es un bien relacional que protegemos y cuidamos.

De hecho es verdad que el principal ámbito para los afectos y las vinculaciones constitutivas es la familia. Sin embargo, no es posible olvidar que durante nuestra experiencia escolar — en particular a la secundaria - se van a consolidar amistades para toda la vida, construir nuestra identitad de manera más conciente, reflexionar sobre problemas y cuestiones complejas. Todos estos acontecimientos brotan de un bumus relacional, comunitario, que incide non solo de manera decisiva en el aprendizaje, sino sobre todo en el destino personal. De hecho, cuando las relaciones humanas y los afectos en la escuela son ignorados o resultan problemáticos, eso obstaculiza el aprendizaje y a raíz de ello el alumno suele buscar otra escuela. Entonces el problema nada tiene que ver con lo académico, pero hay relaciones y afectos escolares que bloquean la mejora personal.

Las ideas de Sandel pueden ayudar a reconsiderar y transformar el sistema escolar, relativizando su dimensión organizativa e instrumental ${ }^{9}$ por un lado; y, por el otro, subrayando la repercusión en el perfeccionamiento y el aprendizaje de las vinculaciones y afectos que son constitutivos, y por ello determinantes. En tal caso, todos los miembros de la comunidad escolar tendrán la misión de cuidar y proteger los vínculos educativamente positivos, que ciertamente permiten crear y compartir una autentica experiencia de vida buena en común. Por supuesto, con todas sus dificultades e incertitumbres, pero siempre rumbo hacia el bien que nos une y nos permite perseguir la más elevada humanidad: el deso de donar y donarse. Consagrarse al bien educativo de los alumnos y alumnas (misión ético-educativa del profesor ${ }^{10}$ ), al bien de compañeros/as de clase (tarea propia de los estudiantes), al bien común ${ }^{11}$ de toda escuela (cometido esencial del director y su equipo).

9. Afirma Sandel (2013, pp. 16-17): «Recompensar a los niños para que lean libros puede moverles a leer más, pero puede también inducirles a considerar la lectura como un trabajo y no como una satisfación personal».

10. Como recuerda Ibañez-Martín (2017, p. 39), hay una fuerte vinculación entre la actividad educativa del docente y la moralidad.

11. A este respecto, como observa agudamente en uno de sus últimos trabajos Sandel (2020), el desmedido énfasis en la meritocracia, también en el ámbito escolar, acaba siendo corrosivo para el bien 
Una vez más, Michael Sandel (2010, pp. 284-285) nos recuerda que, en la vida y la mejora personal, no todo depende de nuestras elecciones y preferencias, y aún menos es el resultado de un simple vínculo contractual y legislativo. De hecho, por ejemplo, la solidaridad y la lealtad son virtudes que no se explican únicamente en virtud de un contrato o un acuerdo. Y todavía menos si las vemos tan solo como el resultado de una simple opción personal (puesto que, por ejemplo, podríamos muy bien escoger no ser solidarios, ni leales). Por el contrario, son solo comprensibles si nos consideramos parte de una social a la cual, sin necesidad de vínculos contractuales y no siempre bajo el impulso de preferencias o elecciones personales y autorreferenciales, sentimos pertenecer o con la cual comprendemos que tenemos deberes y obligaciones.

Así pues, en virtud de cuanto acabamos de señalar, un profesor podrá comprometerse, por el bien común educativo de sus alumnos, a renunciar a cuanto estos se hallen dispuestos a agradecerle o reconocerle por el trabajo que llevo a cabo en su favor. Además, en su quehacer educativo habitual, no se guiará únicamente por lo que su libertad de elección le permitiría escoger. Por ejemplo, muy bien podría limitarse a explicar su materia y cumplir con las tareas burocráticas ligadas a su cargo. Sin embargo, también puede reconocer que tiene una obligación aún mayor de procurar contribuir a la mejora ética a sus estudiantes, aun cuando desde el punto de vista legal, nadie podría obligarlo a perseguir con empeño dicha meta formativa. Este ejemplo permite comprender cómo solo el hecho de estar dentro de una comunidad vuelve posible y al mismo tiempo viables ciertas dimensiones de la educación. ${ }^{12}$

Llegados a este punto, se plantea un nuevo interrogante: ¿Cómo logrará la comunidad educativa escolar realizar su misión educativa sin caer en la manipulación y la homologación? De hecho, como nos recuerda Bianca Thoilliez (2019, p. 301) en una vida lograda "la percepción subjetiva de dicho logro vital, depende del reconocimiento y, por otro lado, que un individuo solo se percibirá a sí mismo como miembro de la comunidad en la medida que se sienta reconocido en una serie de aspectos de su personalidad».

\footnotetext{
común. De hecho la meritocracia genera una nociva complacencia en los triufadores e impone un muy duro castigo a los derrotados.

12. Como explica Sandel (2010, p. 248), «el punto débil de la concepción liberal de la libertad está muy ligado a lo que la vuelve atractiva: si nos concebimos a nosotros mismos comos sujetos libres e independientes, sin ningún vínculo ético que no hayamos escogido, no podemos justificar toda una serie de obligaciones políticas y morales que en la práctica aceptamos y además aprobamos. Entre estos deberes se cuentan la solidaridad y la lealtad. [...], exigencias morales provenientes de la apertura a la comunidad y a las tradiciones que conforman nuestra identidad. Si no nos consideramos sujetos afectados por determinadas obligaciones, por exigencias éticas no voluntarias, resulta muy difícil explicar esos aspectos de nuestra experiencia moral y política».
} 
Por desgracia, a lo largo de la historia, muchas comunidades humanas, incluso ciertas familias, han sembrado la violencia y la intolerancia. De hecho, la influencia de la comunidad, ¿no implica en ocasiones una cierta forma de "violencia»? ¿Al influir en él, no se lo manipularía, si se lo constriñe a ir allí donde él no quiere ir, forzando así su libertad y violando su intimidad?

Es posible, no obstante, evitar este escollo cuando la comunidad, al acompañarme y ayudarme, me permite hallar y poseer el bien constitutivo de mi vida. En el proximo apartado, el último, trataremos esta cuestión.

\section{ESCUELA, COMUNIDAD Y OPCIONES RADICALES}

Taylor nos recuerda que cada persona tiene marcos de referencia vitales que le permiten actuar. Realiza un juicio profundo (strong evaluation) en virtud del cual jerarquiza los bienes y los valores, y en particular, considera que «un cierto modo de vivir o pensar es incomparablemente más elevado que los demás disponibles» (Taylor, 1993, p. 31).

Esta elección permite comprender y asumir la respuesta a las preguntas más radicales: ¿Quién soy? ¿Dónde estoy? ¿Qué es bueno para mí? Interrogantes que pueden generar también esta otra: ¿A dónde voy? (Salerno, 2017, p. 38). Por eso, la definición de la propia identidad ha de partir de un delicado proceso de evaluación, que permite juzgar los bienes y los valores, y también definir el «entorno» que nos puede ayudar a convertirnos en humanos morales. Sin embargo, para lograr tal cosa, explica Taylor (1993), tenemos que decidir cuál es nuestro «bien constitutivo»: aquel bien que nos permite descubrir el sentido más profundo de nuestra vida (pp. 127-128), sin el cual esta perdería su sentido y su significado.

El educador contribuye decisivamente a su formación si acompaña y orienta a alumnos en su camino de discernimiento, categorización y juicio de los valores que van a orientar sus vidas. En tal caso, la comunidad escolar no los manipula con el fin de determinar sus convicciones, descuidando su singularidad, pues respeta ese íntimo proceso de evaluación en el que se define su identidad. Puede occurir que pueden convivir en ella convicciones y articulaciones morales diversas, pero una escuela vivida como comunidad no debe pretender homologar convicciones morales de todos los alumnos, sino facilitar un discernimiento compartido, a un tiempo identitario y ético, que todos procuran impulsar y perfeccionar.

La tarea educativa del docente depende de su capacidad para fomentar, a través del diálogo, el descubrimiento por parte de los alumnos de lo que perciben implicitamente como éticamente justo, pero permitiendo, al mismo tiempo, el despliegue de la originalidad inherente a cada sujeto. Esta originalidad, conviene destacarlo, se manifiesta solo en el diálogo fructífero con una persona de referencia educativa. Para ser conscientes de nuestra unicidad personal necesitamos, imperiosamente, encontrarnos con los demás, por ejemplo, con un profesor que, en el curso del 
diálogo y a la discusión, nos ayude a conceptualizar y orientar el proceso educativo al cual estamos llamados.

La virtualidad formativa de la función docente no pasa por establecer un plan moral inflexible, al que el alumno debe conocer y al cual ha de adaptarse. Por el contrario, hay que inculcar en el estudiante la firme convicción de que existe un horizonte de sentido — de valores e intencional, auténtico y personal—, que bemos de defender y asumir en todas nuestras acciones y no solo en momentos de conveniencia u oportunidad personal.

De hecho, es precisamente un deber educativo esencial de la escuela fomentar en los estudiantes un proceso de mejorar personal en virtud del cual puedan, no solo redescubrir y valorar su peculiar identidad, sino también aportar su singular contribución al entorno social, laboral y familiar donde que están abocados a asumir tareas y responsabilidades. Este camino de autoformación implica necesariamente confrontarse con los valores morales, estrechamente asociados a todo proceso de discernimiento, pero también tiene una dimensión práctica, el compromiso con acciones virtuosas que ciertamente se pueden cultivar y promover en una escuela vivida como comunidad.

Para madurar necesitamos finalidades que nos orienten y nos permitan identificar y escoger la vida buena, que ha de ser el horizonte de referencia, y también la meta que buscamos en cuanto seres humanos educables. Al hombre no le basta solo vivir, sino vivir bien, como nos ha enseñado Aristotelés, pero la vida buena —que «se alcanza desde el saber experiencial y práctico» (López-Jurado \& Kim, 2013, p. 327) - implica comprender y apreciar los pilares que dan sentido a nuestra actividad como profesionales de la educación, y sobre todo como seres humanos. Es así como tal articulación racional de nuestras convicciones morales, fruto de la strong evaluation, adquiere forma y consistencia a luz de ese horizonte de la vida buena, del bien de la persona y del bien moral con el que se hallan en constante relación y diálogo.

Sin embargo, solo podremos captarlos si contamos con una comunidad educativa que nos acoja, nos proteja y nos ayude a comprenderles mejor, e incluso en ciertos a casos a descubrirlos. Si la escuela se concibe de este modo, podrá llevar a cabo su insustituible función —o mejor misión- educativa.

\section{REFERENCIAS BIBLIOGRÁFICAS}

Amilburu, M. G., Bernal, A. y González Martín, M. R. (2018). Antropología de la educación. La especie educable. Síntesis.

Baldacci, M. (2019). La scuola al bivio. Mercato o democrazia?. Franco Angeli.

Balduzzi, E. (2012). La pedagogia del bene comune e l'educazione alla cittadinanza. Vita e Pensiero.

Balduzzi, E. (2015). Liderazgo educativo del profesor en el aula y la personalización educativa. Revista Española de Pegagogía, 73(260), 115-129. https://revistadepedagogia.org/ 
lxxiii/no-260/liderazgo-educativo-del-profesor-en-el-aula-y-la-personalizacion-educativa/101400010438/

Balduzzi, E. (2018). "Sono io il custode di mio fratello». Emozioni e affetti nella scuola vissuta come comunità educativa. Vita e Pensiero.

Bertagna, G. (2020). Vittorino Chizzolini e la pedagogia della scuola. En D. Simeone y M. Busi (Eds.), Vittorino Chizzolini e i giovani. Amare, educare, testimoniare (pp. 86-122). Studium.

Biesta, G. (2012). Philosophy of Education for the Public Good: Five challenges and an agenda. Educational Philosophy and Theory, 46(6), 581-593. https://doi.org/10.1111/j.14695812.2011.00783.x

Consejo de Europa (2018). Recomendación del Consejo del 22 de mayo del 2018 sobre las competencias clave para el aprendizaje permanente. https://eur-lex.europa.eu/legalcontent/IT/TXT/PDF/?uri=CELEX:32018H0604(01)

Ferrara, A. (1992). Comunitarismo e liberalismo. Editori Riuniti.

Genovesi, G. (2020). La scuola un tesoro da salvare: è il primo dovere di uno Stato di diritto. Ricerche Pedagogiche, 214, 5-26. https://www.edizionianicia.it/docs/RP/214-2020/01-\%20 Genovesi\%20C.pdf

Hargreaves, A., \& Shirley, D. (2009). The fourth way: The inspiring future of educational change. Corwin.

Iafrate, R., \& Bertoni, A. (2010). Gli affetti. Dare senso ai legami familiari e sociali. La Scuola.

Ibáñez-Martín, J.A. (2017). Horizontes para los educadores. Las profesiones educativas y la promoción de la plenitud humana. Dykinson.

López-Jurado, M., y Kim, S. (2013). El aprendizaje moral y la vida buena. Revista Española de Pedagogía, 71(255), 327-341. https://revistadepedagogia.org/lxxi/no-255/el-aprendizajemoral-y-la-vida-buena/101400010330/

Malaguti, E. (2016). Educatori, insegnanti e giovani. Il ruolo della scuola e dei gruppi educativi pomeridiani nella promozione di processi di resilienza e inclusione. L'integrazione scolastica e sociale, 15(1), 11-24.

Magni, F. (2020). Introduzione. Per una rinnovata pedagogia delle scuole. Formazione, lavoro, persona, 30, 6-9.

Mari, G. (2013). Educazione come sfida della libertà. La Scuola.

Mari, G. (2014). Scuola e sfida educativa. La Scuola.

Mari, G. (2017). Il docente come educatore: un contributo fondamentale alla qualità delle scuola. Edetania, 52, 107-124. https://revistas.ucv.es/index.php/Edetania/article/view/324

Mari, G. (2018). Competenze educative e servizi alla persona. Studium.

Maritain, J. (2001). Per una filosofia dell'educazione. La Scuola.

Masschelein, J. y Simons, M. (2014). Defensa de la escuela. Una cuestión pública. Miño y Dávila.

Mincu, M. E. (2007). L'educazione non neutrale. La pedagogia dopo la svolta communitarians. SEI.

Mínguez Vallejo, R. (2010). La escuela hoy en la encrucijada. Hacia otra educación desde la ética de E. Lévinas. Teoría de la educación, 22(2), pp. 43-61. https://doi.org/10.14201/8294 
Miur. (2020a). Emergenza sanitaria da nuovo Coronavirus. Prime indicazioni operative per le attività didattiche a Distanza. https://www.itsdallachiesa.edu.it/emergenza-sanitariada-nuovo-coronavirus-prime-indicazioni-operative-per-le-attivita-didattiche-a-distanza/

Miur. (2020b). Adozione del Documento per la pianificazione delle attività scolastiche, educative e formative in tutte le Istituzioni del Sistema nazionale di Istruzione per l'anno scolastico 2020/2021. https://www.istruzioneer.gov.it/wp-content/uploads/2020/06/ DM-ADOZIONE-PAINO-SCUOLA-2020-2021.pdf.pdf

Naval, C. (2000). Educar ciudadanos. La polémica liberal-comunitarista en educación. EUNSA. Nussbaum, M. (2004). L'intelligenza delle emozioni. Il Mulino.

Presidenza della Repubblica italiana, Intervento del Presidente della Repubblica italiana Sergio Mattarella all'inaugurazione dell'anno scolastico 2020/2021. https://www.quirinale.it/ elementi/50200

Puig, J. M. ${ }^{\text {a }}$ (2012). La cultura moral como Sistema de prácticas y mundo de valores. En J. M. Puig (Coord.), Cultura moral y educación (pp. 87-105). Graó.

Rossi, B. (2002). Pedagogia degli affetti. Laterza.

Salerno, V. (2017). Taylor e MacIntyre. Una mappa con i "punti di capitone» A e E di APE. En V. Salerno (Ed.), Non di solo io. Pratiche, educazione e vita buona tra Charles Taylor e Alasdair MacIntyre (pp. 11-94). Libreriauniversitaria.it.

Sandel, M. J. (1994). Il liberalismo e i limiti della giustizia. Feltrinelli.

Sandel, M. J. (2010). Giustizia. Il nostro bene comune. Feltrinelli.

Sandel, M. J. (2013). Quello che i soldi non possono comprare. I limiti morali del mercato. Feltrinelli.

Sandel, M. J. (2020). La Tiranía del Mérito. ¿Qué ha sido del bien común? Penguin Random House.

Sahlberg, P. (2011a). Finnish lessons: What can the world learn from educational change in Finland. Teachers College Press.

Sahlberg, P. (2011b). The fourth way of Finland. Journal of Educational Change, 12(2), 173-185. https://doi.org/10.1007/s10833-011-9157-y

Sandrone, G. (2018). La competenza personale fra formazione e lavoro. Studium.

Sergiovanni, T. (2000). Costruire comunità nelle scuole. LAS.

Strike, K. A. (2000). School as Communities: Four Metaphors, Three Models, and a Dilemma or Two. Journal of Philosophy of Education, 34(4), 617-642. https://doi.org/10.1111/14679752.00198

Taylor, Ch. (1985). The Nature and Scope of Distributive Justice. En Ch. Taylor, Philosophy and the Human Sciences. Philosophical Papers (pp. 289-317). Cambridge University Press.

Taylor, Ch. (1989). Cross-Purposes: the Liberal-Communitarian Debate. En N. Rosemblum (Ed.), Liberalism and the Moral Life (pp. 159-182). Harvard University Press.

Taylor, Ch. (1993). Radici dell'io. La costruzione dell'identità moderna. Feltrinelli.

Thoilliez, B. (2019). Vindicación de la escuela como espacio para el desarrollo de experiencias democráticas: aproximación conceptual a las prácticas morales de reconocimiento y respeto. Educación XX1, 22(1), 295-314. https://doi.org/10.5944/educxx1.21657

Trilla Bernet, J. (2004). Los alrededores de la escuela. Revista Española de Pedagogía, 62(228), 305-324. https://revistadepedagogia.org/lxii/no-228/los-alrededores-de-laescuela/101400010510/ 
Triani, P. (2020). Il rapporto tra scuola e politica. Tre campi di studio per la pedagogia. Encyclopaideia-Journal of Phenomenology and Education, 24(56), 43-49.

Ulivieri, S. (2018). Costruire una nuova democrazia tra conoscenza e solidarietà. Ruolo dell'educazione. En S. Ulivieri, L. Binanti, S. Colazzo \& M. Piccinno (Eds.), Scuola, Democrazia, Educazione. Formare ad una nuova società della conoscenza e della solidarietà (pp. XXI-XXIX). Pensa (e-book). https://www.siped.it/wp-content/uploads/2018/03/2017Convegno-Siped-Leece-CD-ROM.pdf 